\title{
Cochrane
}

Cochrane Database of Systematic Reviews

\section{Adverse effects of immunotherapies for multiple sclerosis: a network meta-analysis (Protocol)}

Tramacere I, Benedetti MD, Capobussi M, Castellini G, Citterio A, Del Giovane C, Frau S, GonzalezLorenzo M, La Mantia L, Moja L, Nuzzo S, Filippini G

Tramacere I, Benedetti MD, Capobussi M, Castellini G, Citterio A, Del Giovane C, Frau S, Gonzalez-Lorenzo M, La Mantia L, Moja L, Nuzzo S, Filippini G.

Adverse effects of immunotherapies for multiple sclerosis: a network meta-analysis.

Cochrane Database of Systematic Reviews 2016, Issue 5. Art. No.: CD012186.

DOI: 10.1002/14651858.CD012186.

www.cochranelibrary.com 
TABLE OF CONTENTS

HEADER . . . . . . . . . . . . . . . . . . . . . . . . . . . . . . . . . . . . . . . . 1

ABSTRACT . . . . . . . . . . . . . . . . . . . . . . . . . . . . . . . . . . . . . . 1

BACKGROUND . . . . . . . . . . . . . . . . . . . . . . . . . . . . . . . . . . . .

OBJECTIVES . . . . . . . . . . . . . . . . . . . . . . . . . . . . . . . . . . . . . . . 5

METHODS . . . . . . . . . . . . . . . . . . . . . . . . . . . . . . . . . . . . . . .

ACKNOWLEDGEMENTS . . . . . . . . . . . . . . . . . . . . . . . . . . . . . . . . . . . . . . . . . .

REFERENCES . . . . . . . . . . . . . . . . . . . . . . . . . . . . . . . . . . . . . . . . 10

APPENDICES . . . . . . . . . . . . . . . . . . . . . . . . . . . . . . . . . . . . . 14

CONTRIBUTIONS OF AUTHORS . . . . . . . . . . . . . . . . . . . . . . . . . . . . . . . . . . . . . . .

DECLARATIONS OF INTEREST . . . . . . . . . . . . . . . . . . . . . . . . . . . . . . . 15

SOURCES OF SUPPORT . . . . . . . . . . . . . . . . . . . . . . . . . . . . . . . . . . . . . . . . 


\title{
Adverse effects of immunotherapies for multiple sclerosis: a network meta-analysis
}

\author{
Irene Tramacere ${ }^{1}$, Maria Donata Benedetti ${ }^{2}$, Matteo Capobussi ${ }^{3}$, Greta Castellini ${ }^{3}, 4$, Antonietta Citterio ${ }^{5}$, Cinzia Del Giovane ${ }^{6}$, Serena \\ Frau $^{7}$, Marien Gonzalez-Lorenzo ${ }^{3}$, Loredana La Mantia ${ }^{8}$, Lorenzo Moja ${ }^{3}{ }^{9}$, Sara Nuzzo ${ }^{10}$, Graziella Filippini ${ }^{11}$ \\ ${ }^{1}$ Neuroepidemiology Unit, Fondazione IRCCS Istituto Neurologico Carlo Besta, Milan, Italy. ${ }^{2}$ UOC Neurologia B - Policlinico \\ Borgo Roma, Azienda Ospedaliera Universitaria Integrata, Verona, Italy. ${ }^{3}$ Department of Biomedical Sciences for Health, University of \\ Milan, Milan, Italy. ${ }^{4}$ Unit of Clinical Epidemiology, IRCCS Galeazzi Orthopaedic Institute, Milan, Italy. ${ }^{5}$ Scientific Direction, IRCCS \\ National Neurological Institute C. Mondino, Pavia, Italy. ${ }^{6}$ Italian Cochrane Centre, Department of Diagnostic, Clinical and Public \\ Health Medicine, University of Modena and Reggio Emilia, Modena, Italy. ${ }^{7}$ Brighton and Hove, UK. ${ }^{8}$ Unit of Neurorehabilitation \\ - Multiple Sclerosis Center, IRCCS. Santa Maria Nascente - Fondazione Don Gnocchi, Milan, Italy. ${ }^{9}$ Clinical Epidemiology Unit, \\ IRCCS Galeazzi Orthopaedic Institute, Milan, Italy. ${ }^{10}$ Fondazione IRCCS. Istituto Neurologico Carlo Besta, Milan, Italy. ${ }^{11}$ Scientific \\ Direction, Fondazione IRCCS. Istituto Neurologico Carlo Besta, Milan, Italy
}

Contact address: Irene Tramacere, Neuroepidemiology Unit, Fondazione IRCCS Istituto Neurologico Carlo Besta, Via Giovanni Celoria, 11, Milan, 20133, Italy. irene.tramacere@istituto-besta.it.

Editorial group: Cochrane Multiple Sclerosis and Rare Diseases of the CNS Group.

Publication status and date: New, published in Issue 5, 2016.

Citation: Tramacere I, Benedetti MD, Capobussi M, Castellini G, Citterio A, Del Giovane C, Frau S, Gonzalez-Lorenzo M, La Mantia L, Moja L, Nuzzo S, Filippini G. Adverse effects of immunotherapies for multiple sclerosis: a network meta-analysis. Cochrane Database of Systematic Reviews 2016, Issue 5. Art. No.: CD012186. DOI: 10.1002/14651858.CD012186.

Copyright (C) 2016 The Cochrane Collaboration. Published by John Wiley \& Sons, Ltd.

\begin{abstract}
A B S T R A C T
This is the protocol for a review and there is no abstract. The objectives are as follows:

To compare adverse effects of immunotherapies for people with multiple sclerosis (MS) or clinically isolated syndrome (CIS), and to rank these treatments according to their relative risks of adverse effects.
\end{abstract}

\section{B A C K G R O U N D}

\section{Description of the condition}

\section{General view}

Multiple sclerosis (MS) is a chronic disease of the central nervous system (CNS) that affects mainly young adults - two to three times more frequently women than men - and causes significant disability two or more decades after onset. Neurological signs and symptoms reflect dissemination of inflammatory lesions (plaques) through different areas of the CNS, including optic nerve, spinal cord, brainstem, cerebellum and cerebral hemispheres. The initial clinical course is characterised by exacerbations and remissions, with complete or incomplete recovery reported in more than $85 \%$ of cases, and a primary progressive course noted in $10 \%$ to $15 \%$ (Compston 2008). Disease severity is variable, with some patients accumulating a low degree of disability during their lifetime, and others experiencing within a short time a rapidly aggressive clinical 
course.

\section{Pathogenesis}

The pathological hallmark of MS consists of focal inflammation, demyelination, axonal degeneration and gliosis, interplaying in variable degrees over different stages of the disease. It is still debated whether inflammation, clinically expressed by relapses, and neurodegeneration, which causes progression of disability, are sequential or independent processes (Compston 2008; Hutchinson 2015; Louapre 2015; Weinshenker 1989). A heterogeneous population of mediators of the immune system, such as T cells, B cells, macrophages, cytokines, chemokines and complement, is involved in inflammatory demyelinating processes, while activation of microglia, chronic oxidative stress, mitochondrial damage in axons and age-related iron accumulation drive neurodegeneration (Brück 2005; Friese 2014).

\section{Etiology}

Multiple sclerosis is believed to be an immune-mediated disease triggered by environmental factors in genetically susceptible individuals (Compston 2008). Epstein-Barr virus infection, low vitamin D levels and smoking are the environmental factors most consistently associated with this disease (Ascherio 2013). The genetic contribution to MS development is not yet completely elucidated, although to date more than 100 loci have been associated with MS genetic susceptibility, many related to immune function genes, and the HLA-DR1 locus within the major histocompatibility complex (MHC) has shown the strongest effect (Hollenbach 2015; Simpson 2015). The frequency of MS increases with increasing latitude in both northern and southern hemispheres, with incidence estimates ranging from 1 to $10 / 100,000$ population per year in different places in the world, and with Europe and North America expressing highest prevalence of the disease ( $>100$ cases per 100,000 population) (Atlas of MS 2013).

\section{Phenotype classification}

Clinical subtypes of MS defined in 1996 by a consensus paper include relapsing-remitting MS (RRMS), secondary-progressive MS (SPMS), primary-progressive MS (PPMS) and progressive-relapsing MS (PRMS) (Lublin 1996). A recent revision of this classification incorporates disease activity on the basis of clinical relapse or imaging findings and progression of disability as disease course descriptors, eliminating the prior category of PRMS (Lublin 2014). This revision also includes clinically isolated syndrome (CIS), already defined by McDonald diagnostic criteria (2001) as the first clinical presentation of MS in which the criterion of dissemination over time has not yet been fulfilled (Lublin 2014).

\section{Diagnostic criteria}

Over past decades, MS was clinically diagnosed when two separate attacks (dissemination in time - DIT) were observed in at least two different sites (dissemination in space - DIS) of the CNS, with any better explanation excluded (Poser 1983). In the McDonald diagnostic criteria of 2001 and 2005, magnetic resonance imaging (MRI) was incorporated to define DIS and DIT, allowing earlier diagnosis of MS (McDonald 2001; Polman 2005). According to the most recent revision (Polman 2011), MS may be diagnosed with a single clinical episode when a single brain MRI shows both asymptomatic gadolinium-enhancing and non-enhancing lesions.

\section{Clinical course}

Natural history studies have reported a transition to a secondary progressive course in some $80 \%$ of RRMS patients within 20 to 25 years of onset when neurodegenerative processes prevailed over inflammatory changes, with similar age at onset of progression and disease evolution during the progressive phase in SPMS and PPMS (Confavreux 2006; Kremenchutzky 2006; Scalfari 2014; Weinshenker 1989). Progressive phase delay, prevention and attenuation are key therapeutic targets in MS (Scalfari 2010). Median survival from disease onset may reach five decades, with age at death beyond 75 and life expectancy reduced by seven to 14 years compared with the general population (Scalfari 2013).

\section{Therapy}

Although neuroprotective drugs against axonal loss and degeneration are not available, several drugs have been tested and approved over the past 20 years for RRMS treatment on the basis of the immune-mediated mechanism of the disease in an attempt to reduce active inflammatory manifestations (relapses) with the goal of delaying conversion of CIS in definitive MS or the transition to a secondary progressive course. No treatment for PPMS has actually been approved.

Critical points in recent MS randomised clinical trials (RCTs) consist of inclusion of people at earlier stages of disease with lower disease activity compared with historical RCTs, limited use of placebo for ethical reasons and short trial duration to demonstrate drug efficacy, so that safety outcomes become manifest in postmarketing observations.

\section{Description of the intervention}

We will consider all immunotherapies that are used, whether approved or off-label, or are currently under marketing authorisation or investigation for people with MS or CIS. 


\section{Approved}

Beta interferons (Betaferon/Betaseron ${ }^{\circledR} ;$ Extavia $^{\circledR} ; \quad$ Rebif ${ }^{\circledR}$; Avonex ${ }^{\circledR}$ ) and glatiramer acetate $\left(\right.$ Copaxone ${ }^{\circledR}$ ) were the first agents approved by national regulatory agencies (EMEA 1997; EMEA 1998; EMEA 2002; FDA 1993; FDA 1996; FDA 2002; FDA 2003). These medications are administered subcutaneously, except for beta interferon 1a (Avonex ${ }^{\circledR}$ ), which is administered by intramuscular injection.

Mitoxantrone (Novantrone ${ }^{\circledR}$ ) was approved in 2000 in the USA (FDA 2000), Europe and other countries for treatment of patients with RRMS and progressive MS. A short intravenous infusion is given every three months.

Natalizumab (Tysabri ${ }^{\circledR}$ ), approved for RRMS (EMA 2006; FDA 2006), is administered by intravenous infusion at a dose of 300 mg every four weeks.

Fingolimod $\left(\right.$ Gilenya $^{\circledR}$ ), approved for RRMS (EMA 2011; FDA $2010)$, is given as a $0.5 \mathrm{mg}$ oral dose once daily.

Teriflunomide (Aubagio ${ }^{\circledR}$ ), approved for RRMS (EMA 2013a; FDA 2012), is given as a 7 or $14 \mathrm{mg}$ oral dose once daily.

Dimethyl fumarate (Tecfidera ${ }^{\circledR}$ ), approved for RRMS (EMA 2014a; FDA 2013a), is given as a $240 \mathrm{mg}$ oral dose twice daily. Alemtuzumab (Lemtrada ${ }^{\circledR}$ ), approved for RRMS (EMA 2013b; FDA 2014a), is administered intravenously in two annual treatment courses - the first as a $12 \mathrm{mg}$ dose daily on five consecutive days (60 mg total dose), and the second, 12 months later, on three consecutive days (36 $\mathrm{mg}$ total dose).

Peginterferon beta-1a $\left(\right.$ Plegridy $^{\circledR}$ ), approved for RRMS (EMA 2014b; FDA 2014b), is given by subcutaneous injection at a dose of 125 micrograms every 14 days.

Cladribine (Movectro ${ }^{\circledR}$ ) was approved in Russia and Australia for RRMS in 2010 (Murphy 2010), but it was not approved by the European Medicines Agency (EMA) (EMA 2010) and the Food and Drug Administration (FDA) in 2011 because of a suspected increase in cancer risk that has not been confirmed by results of a meta-analysis of trials (Pakpoor 2015). Cladribine was investigated in two trials (Giovannoni 2010; Leist 2014): It was given as tablets at a cumulative dose of $3.5 \mathrm{mg} / \mathrm{kg}(0.875 \mathrm{mg} / \mathrm{kg}$ per course, given over four or five consecutive days, for two courses during the first 48 weeks, then two courses of cladribine $0.875 \mathrm{mg} / \mathrm{kg}$ during the second 48 weeks) or $5.25 \mathrm{mg} / \mathrm{kg}(0.875 \mathrm{mg} / \mathrm{kg}$ per course for four courses during the first 48 weeks, then two courses during the second 48 weeks).

\section{Used off-label}

Azathioprine has been used for the treatment of patients with MS in many countries on the basis of placebo-controlled RCTs published more than two decades ago. However, since beta interferons were approved, azathioprine is no longer recommended as firstline therapy (Goodin 2002). It is taken orally daily as a 2 or $3 \mathrm{mg} /$ kg tablet.
Intravenous immunoglobulins have been used for people with severe and frequent relapses, for whom other treatments were contraindicated (Association of British Neurologists 2005).

Rituximab has been evaluated in two trials (Hauser 2008; Hawker 2009). In one trial that included participants with RRMS (Hauser 2008), study authors found beneficial effects on clinical and MRIvisualised disease activity that were maintained over 48 weeks; in the other trial of participants with PPMS (Hawker 2009), study authors found no significant effects on time to confirmed disease progression. This drug is administered intravenously.

Methotrexate is commonly prescribed in general practice to control autoimmune diseases, including neuromyelitis optica; it has been used since 1996 for MS (Goodkin 1996), mainly progressive MS; it is taken orally as $7.5 \mathrm{mg}$ weekly (with $1 \mathrm{mg}$ daily folic acid supplementation) (Gray 2004).

Cyclophosphamide is an alkylating agent of DNA used for the treatment of patients with autoimmune disorders. It has been administered to patients with MS since 1991 by various schedules as $1 \mathrm{~g}$ i.v. over three days, or 400 to $500 \mathrm{mg} / \mathrm{d}$ i.v. over five days alone or along with adrenocorticotropin hormone (ACTH) or plasma exchange, or it has been given orally at $2 \mathrm{mg} / \mathrm{kg} / \mathrm{d}$ (La Mantia 2007).

Long-term corticosteroids have been proposed for the treatment of patients with MS since 1961, with controversial results. They have been administered by different schedules as pulsed periodic high-dose methylprednisolone or oral continuous low-dose prednisolone (Ciccone 2008).

\section{Currently under marketing authorisation or investigation}

Daclizumab (Zenapax ${ }^{\circledR}$ ) is administered by subcutaneous or intravenous injection. Review processes by EMA and FDA are ongoing.

Laquinimod (Nerventra ${ }^{\circledR}$ ) was investigated in two phase 3 trials for treatment of patients with RRMS (Comi 2012; Vollmer 2014). An oral dose of $0.6 \mathrm{mg}$ daily has been used in trials. This drug received a negative response from the EMA for use in treating patients with RRMS (EMA 2014c). Additional studies on laquinimod in people with RRMS are ongoing.

Ocrelizumab is under development for treatment of patients with RRMS and PPMS, and clinical trials are ongoing (Kappos 2011). It is administered by intravenous injection.

\section{How the intervention might work}

The harm profile of an intervention is strictly related to its mechanism of action, its modality of administration and pharmacokinetic, pharmacodynamic and possibly pharmacogenetic aspects of drug response (Goodman 2006).

According to the International Conference on Harmonisation of Technical Requirements for Registration of Pharmaceuticals for 
Human Use (ICH 2015), adverse events (AEs) are classified in terms of system organ class (SOC), that is, by identifying the anatomical or physiological system affected by the AE itself. Immunotherapies for MS belong to different pharmacological categories, have different modalities of administration (by intramuscular or subcutaneous injection, by infusion or by mouth) and have different metabolism; although all target the immune system, they are characterised by different effects, as follows: (1) immunomodulation (interferon beta-1b, interferon beta-1a (Avonex, Rebif), glatiramer acetate, pegylated interferon beta-1a, immunoglobulins, dimethyl fumarate, laquinimod); (2) systemic immunosuppression, inducing a reduction in activation or efficacy of the immune system through cytostatic or cytotoxic effects (mitoxantrone, methotrexate, cyclophosphamide, long-term corticosteroids, cladribine, azathioprine, teriflunomide); and (3) selective immunosuppression, as with monoclonal antibodies or biological agents directed towards exactly defined antigens (natalizumab, fingolimod, alemtuzumab, daclizumab, rituximab, ocrelizumab). These aspects must be considered when the safety profile of a drug is determined because safety is usually a consequence of the drug's primary pharmacological effect.

We might classify main types and the etiopathogenesis of AEs of MS immunotherapies according to the Medical Dictionary for Regulatory Activities System Organ Classes (MedDRA SOC), as follows.

- Immune system disorders. All immunotherapies may cause acute or delayed systemic reactions due to allergic response, anaphylaxis, autoimmune disorder, cytokine release syndrome and serum sickness. Such reactions occur in particular during monoclonal antibody treatment (Lycke 2015) but also with immunomodulating agents, such as interferons. The exact process of flu-like interferon syndrome is poorly understood but probably is related to increased endogenous pyrogens such as interleukin-6 (IL-6) and tumour necrosis factor-alpha (TNF- $\alpha$ ) (Martìnez-Càceres 1998). Autoimmune diseases such as thyroiditis, psoriasis and rheumatoid arthritis are more frequent in people treated with immunomodulatory or immunosuppressive drugs than in naive patients (Chouhfeh 2015).

- Blood and lymphatic system disorders. Cytostatic effects or selective antagonism versus critical cell antigens might cause complete or partial myelodepression, or lymphopenia. This latter AE occurs, for example, in fingolimod-treated people, as the result of prevention of egress from secondary lymphoid tissues or following use of alemtuzumab, which selectively causes depletion of $\mathrm{T}$ and $\mathrm{B}$ lymphocytes. The mechanisms of these AEs during immunomodulating therapies (interferons, dimethyl fumarate) remain uncertain.

- Infections and infestations. These might occur during immunosuppressive therapies that impair the immune system and induce immunosurveillance depression. Opportunistic infection such as progressive multi-focal leukoencephalopathy
(PML) in people treated with natalizumab seems to be due to inhibition of effector T-cell trafficking from blood to CNS, which might favour local John Cunningham virus (JCV) replication (Van Assche 2005). PML has also been reported in people treated with fingolimod or dimethyl fumarate, probably resulting from similar causes. Other opportunistic infections such as herpes virus reactivation and tuberculosis are associated with immunosuppressive or immunomodulatory therapies (Williamson 2015).

- Pregnancy, puerperium and perinatal conditions. Pregnancy and foetal damage have been reported with all therapies, although with different severity of harm or risk for reproductive potential and pregnancy category rating (Federal Register 2015). They are probably related to pharmacological effects on DNA and RNA replication (Amato 2015).

- Neoplasms benign, malignant and unspecified. The relative carcinogenicity of each agent (not described until now for immunomodulating agents) is not well understood. Different types of tumours have been found to be related to use of selective or non-selective immunosuppressive drugs such as fingolimod, mitoxantrone and azathioprine, which have been associated with risk of skin carcinoma, leukaemia and myelodysplastic syndrome, respectively (Casetta 2007; Martinelli-Boneschi 2005).

AEs such as hepatic disorders are common to all types of drugs; others seem to be strictly related to a specific compound. Fingolimod causes transient activation of sphingosine-1-phosphate receptor 1 (S1P1) in atrial myocytes, which is associated with a transient reduction in heart rate, while lung hyperreactivity leading to bronchospasms and airway constriction is mediated by S1P1 and sphingosine-1-phosphate receptor 3 (S1P3) activation. Alemtuzumab treatment is associated with risk of secondary autoimmunity due to reconstitution of the lymphocyte repertoire. Dimethyl fumarate-treated people have experienced flushing and gastrointestinal problems, although the causes of these events remain uncertain (Bomprezzi 2015).

Many of these AEs are known and expected on the basis of a drug's mechanism of action and pharmacodynamic aspects; other reactions remain of uncertain origin or appear during long-term monitoring of people. Familiarity with the safety profile of each drug is critical for identification of potential mitigation strategies (Farber 2015).

\section{Why it is important to do this review}

Although consensus indicates that immunotherapies for people with MS may decrease disease activity, uncertainty regarding their relative safety remains. Systematic assessments have not been performed to compare short-, medium- and long-term adverse effects of each immunotherapy versus the others. 


\section{O B J E C T I V E S}

To compare adverse effects of immunotherapies for people with multiple sclerosis (MS) or clinically isolated syndrome (CIS), and to rank these treatments according to their relative risks of adverse effects.

\section{METHODS}

\section{Criteria for considering studies for this review}

\section{Types of studies}

We will include all phase 2 and 3 RCTs that examined one or more of the agents used in MS or CIS and compared them versus placebo or another active agent. We will exclude RCTs in which a drug regimen was compared with a different regimen of the same drug without another active agent or placebo as a control arm.

\section{Types of participants}

We will include participants 18 years of age or older with a diagnosis of MS or CIS according to any accepted diagnostic criteria (Lublin 1996; McDonald 2001; Polman 2005; Polman 2011; Poser 1983). We will include all participants regardless of sex, degree of disability or disease duration.

\section{Types of interventions}

We will include the following immunotherapies (even if they are not licensed in any country) used as monotherapies (i.e. we will exclude combination treatments).

- Interferon beta-1b.

- Interferon beta-1a (Avonex, Rebif).

- Glatiramer acetate.

- Mitoxantrone.

- Natalizumab.

- Fingolimod.

- Teriflunomide.

- Dimethyl fumarate.

- Alemtuzumab.

- Pegylated interferon beta-1a.

- Cladribine.

- Azathioprine.

- Immunoglobulins.

- Rituximab.

- Daclizumab.

- Laquinimod.

- Ocrelizumab.

- Methotrexate.
- Cyclophosphamide.

- Long-term corticosteroids.

We will include regimens as defined in primary studies, irrespective of their dose and treatment duration.

\section{Types of outcome measures}

\section{Primary outcomes}

We will estimate the relative risks of adverse effects at longest follow-up of competing interventions according to the following primary outcomes.

- Number of participants with any (one or more) serious adverse events (SAEs).

- Number of withdrawals due to adverse events (AEs).

We will adopt the following standard definitions of AEs and SAEs: An adverse event is an adverse outcome that occurs while a patient is taking a drug but is not (or is not necessarily) attributable to the drug taken; serious adverse events are adverse events that occur at any dose and result in death or life-threatening events, requirement for hospitalisation or prolongation of existing hospitalisation, persistent or significant disability or congenital anomalies, or are considered medically important (ICH 2015).

\section{Secondary outcomes}

We will estimate the relative risks of adverse effects at longest follow-up of competing interventions according to the following secondary outcomes, as classified by the Medical Dictionary for Regulatory Activities System Organ Classes (MedDRA SOC) (version 18.0) (ICH 2015).

- Cardiac disorders (SAEs and AEs, separately).

- Infections and infestations (SAEs and AEs, separately).

- Administration site conditions (SAEs and AEs, separately).

- Nervous system disorders (SAEs and AEs, separately).

- Psychiatric disorders (SAEs and AEs, separately).

- Gastrointestinal disorders (SAEs and AEs, separately).

- Blood and lymphatic system disorders (SAEs and AEs, separately).

- Hepatobiliary disorders (SAEs and AEs, separately).

- Immune system disorders (SAEs and AEs, separately).

- Pregnancy, puerperium and perinatal conditions.

- Deaths.

- Neoplasms.

We will express all outcomes for each SAE category as percentages of participants with any (one or more) SAEs. We will express all outcomes for each AE category as rates of AEs (i.e. number of AEs divided by person-years of exposure). We expect that person-years of exposure will not be available for each study; therefore, we will 
extract the duration of observation, so that this can be considered as person-years of exposure.

\section{Search methods for identification of studies}

We will search for all possible comparisons among interventions of interest and will apply no language restrictions to the search.

\section{Electronic searches}

The Trials Search Co-ordinator will search the Trials Register of the Cochrane Multiple Sclerosis and Rare Diseases of the CNS Group, which, among other sources, contains trials from:

- Cochrane Central Register of Controlled Trials

(CENTRAL) (2016, most recent issue);

- MEDLINE (PubMed) (1966 to date);

- EMBASE (EMBASE.com) (1974 to date);

- Cumulative Index to Nursing and Allied Health Literature (CINAHL) (EBSCOhost) (1981 to date);

- Latin American and Caribbean Health Science Information Database (LILACS) (Bireme) (1982 to date);

- ClinicalTrials.gov (www.clinicaltrials.gov); and

- World Health Organization (WHO) International Clinical Trials Registry Platform (apps.who.int/trialsearch).

Information on the Trials Register or the Review Group and details of the search strategies used to identify trials can be found in the 'Specialised Register' section within the Cochrane Multiple Sclerosis and Rare Diseases of the CNS Group module.

The keywords that will be used to search for trials for this review are listed in Appendix 1.

\section{Searching other resources}

We will extend the search to other resources, including:

- search of FDA pre-marketing and post-marketing reports providing safety data on all treatments included in this review ( www.fda.gov);

- search of EMA pre-marketing and post-marketing reports providing safety data on all treatments included in this review ( www.ema.europa.eu); and

- search of Australian medicines regulatory authority (the Therapeutic Goods Administration - TGA) pre-marketing and post-marketing reports providing safety data on all treatments included in this review (www.tga.gov.au).

\section{Data collection and analysis}

\section{Selection of studies}

We will use the search strategy described above to obtain titles and abstracts of studies that may be relevant to the review. Two teams of two review authors each (GC and SF; MGL and MC) will independently screen titles and abstracts and will discard studies that are not applicable; however, we will at first retain studies and reviews that might include relevant data or information on trials. Two teams of two review authors each (GC and SF; MGL and $\mathrm{MC)}$ will independently assess the retrieved abstracts and, when necessary, the full text of these studies to determine which studies satisfy the inclusion criteria. We will compare multiple reports of the same study and will use the most comprehensive report. We will not link together multiple publications of the primary study that did not report AEs, and we will exclude true duplicates. We will resolve discrepancies in judgement by discussion with a third review author (IT).

\section{Data extraction and management}

Two review authors (IT and SN) will independently extract data using a pre-defined data extraction form within an Excel spreadsheet. We will resolve disagreements by discussion with a third review author (GF).

\section{Outcome data}

We will extract from each included study the number of participants who:

- had any SAE;

- withdrew because of any AE;

- experienced any specific AE or SAE according to the MedDRA SOC (ICH 2015), as defined in the Types of outcome measures section;

- were randomised; and

- took one or more doses of treatment.

We will extract arm-level data when possible. When arm-level data are not available, we will extract effect sizes.

When data are not reported or are unclear in the primary studies, we will consult reports from FDA, EMA and TGA.

\section{Data on potential effect modifiers}

We will extract from each included study data on the following potential effect modifiers.

- Population: age (range), disease course (CIS, RRMS, SPMS, PPMS and PRMS), disease duration for MS and time since neurological event for CIS (mean if provided or median), EDSS (mean), previous treatment with immunotherapies (no or yes/possible).

- Study design: duration of follow-up.

- Intervention: dose, frequency or duration of treatment.

- Risk of bias: blinding of participants, blinding of outcome assessors, incomplete outcome data. 


\section{Other data}

We will extract from each included study data on the following additional information.

- Study: first author or acronym, number of centres, year of publication, recruitment period, publication type (full-text publication, abstract publication, unpublished data).

- Study design: inclusion criteria, sequence generation, allocation concealment, selective outcome reporting, early termination of trial.

\section{Assessment of risk of bias in included studies}

We will assess the risk of bias of each included study by using the criteria of The Cochrane Collaboration (Higgins 2011). These include random sequence generation, allocation concealment, blinding of participants, blinding of outcome assessors, incomplete outcome data and selective outcome reporting. Another potential risk of bias involves the role of the sponsor. We will explicitly judge the risk of bias of each study on the basis of each criterion and will classify the study as having 'low', 'high', or 'unclear' risk of bias. We will judge incomplete outcome data as showing low risk of bias when numbers and causes of dropouts are balanced (i.e. in the absence of a significant difference) between arms and appear to be unrelated to studied outcomes. We will judge selective outcome reporting as showing low risk of bias when study results include the three outcome categories relevant to the review, that is, SAEs, AEs and withdrawals due to AEs.

To summarise the quality of the evidence, we will consider blinding of participants, blinding of outcome assessors and incomplete outcome data to classify each study as having low risk of bias when we judge all of the selected criteria as having low risk of bias; high risk of bias when we judge at least one criterion among those selected as having high risk of bias; and moderate risk of bias in the remaining cases.

We will assess characteristics associated with monitoring and reporting of adverse events by considering specific factors that may have a large influence on adverse event data. We will evaluate methods of monitoring and detecting adverse events in each primary study: Did researchers actively monitor for adverse events, or did they simply provide spontaneous reporting of adverse events that arose? Did study authors define SAEs according to an accepted international classification and report the number of SAEs? We will report this information in an additional table called 'Assessment of adverse event monitoring'.

Two teams of two review authors each (GC and SF; MGL and $\mathrm{MC}$ ) will assess the risk of bias of each study independently and will resolve disagreements by discussion to reach consensus.

\section{Measures of treatment effect}

\section{Relative treatment effects}

We will estimate, through pairwise meta-analysis, the safety of competing interventions by using the risk or rate ratio (RR) with 95\% confidence intervals ( $95 \%$ CIs) for each outcome. We will present results from network meta-analysis as summary relative effect sizes (RR) with $95 \%$ CIs for each possible pair of treatments.

\section{Relative treatment ranking}

We will estimate ranking probabilities for all treatments at each possible rank for each intervention for each outcome. We will then determine a treatment hierarchy by using the surface under the cumulative ranking curve (SUCRA) and mean ranks (Salanti 2011).

\section{Unit of analysis issues}

Cluster and cross-over trials have not been carried out to evaluate immunotherapies for the treatment of people with MS or CIS.

\section{Studies with multiple treatment groups}

For multi-arm trials, the intervention groups of relevance will be those that could be included in a pairwise comparison of intervention groups, which, if investigated alone, would have met the criteria for inclusion of studies in the review. For example, if we identify a study comparing 'interferon beta versus natalizumab versus interferon beta plus natalizumab', only one comparison ('interferon beta vs natalizumab') addresses the review objective, and no comparison involving combination therapy does so. Thus, the 'interferon beta plus natalizumab' therapy group is not relevant to the review. However, if the study had compared 'interferon beta- 1 b versus interferon beta- 1 a (Rebif) versus interferon beta- 1 a (Avonex)', all three pairwise comparisons of interventions would be relevant to the review. In this case, we would treat multi-arm studies as multiple independent two-arm studies in pairwise metaanalysis and would account for the correlation between effect sizes in multi-arm studies through network meta-analysis.

We will explore whether safety of the agent is modulated by treatment dose as follows. We will consider the network formed from agents administered at different doses (so that each node in the network represents treatment at a different dose). We will transform any specific dose used for each agent into a unique unit of measure - 'dose per week'. We will re-analyse the network and will derive network meta-analysis estimates for each drug and dose. If no important dose-effect relationship is found, we will convert multi-arm trials involving the same agent at different doses versus a control treatment into a single arm by merging doses and summing numbers of events and sample size. Conversely, we will analyse separately different treatment doses with different effects. 


\section{Dealing with missing data}

A likely scenario for assessment of effects of missing data on $\mathrm{AE}$ outcomes (i.e. rates of AEs) is not feasible, and on SAE outcomes is nonsense (i.e. assuming that participants who contributed to missing outcome data had an SAE); therefore, we will perform a sensitivity analysis including only trials with low risk of attrition bias and will discuss the extent to which missing data could alter results/conclusions of the review.

\section{Assessment of heterogeneity}

\section{Assessment of clinical heterogeneity within treatment comparisons}

To evaluate the presence of heterogeneity derived from different characteristics of study participants, we will assess differences in age, MS course, disease duration and EDSS across trials using information reported in the 'Characteristics of included studies' table.

\section{Assessment of transitivity across treatment comparisons}

We will evaluate the assumption of transitivity by comparing potential effect modifiers as reported in the Data extraction and management section, across different pairwise comparisons.

\section{Assessment of reporting biases}

Given that it is not mandatory for investigators to publish results of clinical trials, it is difficult for review authors to obtain an estimate of the number of unpublished trials on MS. We will evaluate the possibility of reporting bias by creating contour-enhanced funnel plots (Peters 2008), which show areas of statistical significance and can help to distinguish reporting bias from other possible reasons for asymmetry. Each study estimates the relative effects of different interventions, so asymmetry in the funnel plot cannot be judged. To account for this, we will use an adaptation of the funnel plot by subtracting from each study-specific effect size the mean derived by meta-analysis of the study-specific comparison and will plot it against the study's standard error (Chaimani 2012; Chaimani 2013). We will employ the comparison-adjusted funnel plot for all placebo-controlled trials and will point out that any asymmetry in the plot indicates the presence of small study effects, not necessarily reporting bias.

\section{Data synthesis}

\section{Methods for direct treatment comparisons}

We will perform conventional pairwise meta-analyses for each primary outcome using a random-effects model for each treatment comparison with at least two studies (DerSimonian 1986).

\section{Methods for indirect and mixed comparisons}

We will perform network meta-analyses using random-effects models within a frequentist setting assuming equal heterogeneity across all comparisons, and we will account for correlations induced by multi-arm studies (Miladinovic 2014; Salanti 2012). These models will enable us to estimate the probability that each intervention will be at each possible rank for each outcome, given the relative effect sizes as estimated in the network meta-analysis. We will summarise the probabilities that a treatment will be at each possible rank by using SUCRAs. By using the cluster analysis technique, we will group treatments according to SUCRA values and will present them in a plot. We will perform network metaanalysis in Stata 13 using the 'mvmeta' command and self programmed Stata routines (Chaimani 2013; Multiple-Treatments Meta-analysis (MTM); White 2011; White 2012).

\section{Subgroup analysis and investigation of heterogeneity}

\section{Assessment of statistical heterogeneity}

\section{Assumptions when heterogeneity is estimated}

As we expect to include few studies (around two to four) in each direct comparison, in standard pairwise meta-analysis we will assume a common heterogeneity variance for all direct comparisons. In network meta-analysis, we will assume a common estimate for the heterogeneity variance across different comparisons.

\section{Measures and tests for heterogeneity}

We will statistically assess the presence of heterogeneity for all direct pairwise comparisons using common $\tau^{2}$ and $\mathrm{I}^{2}$ statistics. Assessment of statistical heterogeneity in the entire network will be based on the magnitude of the heterogeneity variance parameter $\left(\tau^{2}\right)$ estimated by using network meta-analysis models (Jackson 2014).

\section{Assessment of statistical inconsistency}

We will assume that any patient who met the inclusion criteria was, in principle, equally likely to have been randomised to any of the eligible interventions. 


\section{Local approaches for evaluating inconsistency}

To evaluate the presence of inconsistency locally, we will use the loop-specific approach. We will use this method to evaluate the consistency assumption in each closed loop of the network separately as the difference between direct and indirect estimates for a specific comparison within the loop (inconsistency factor) (Veroniki 2013). We then can use the magnitude of the inconsistency factors and their $95 \%$ CIs to infer the presence of inconsistency in each loop. We will assume a common heterogeneity estimate within each loop and will present the results of this approach graphically in a forest plot using the 'ifplot' command in Stata (Chaimani 2013).

\section{Global approaches for evaluating inconsistency}

We will use the 'design-by-treatment' model to evaluate the assumption of consistency across the entire network (Higgins 2012). This method accounts for different sources of inconsistency seen when studies with different designs (two-arm trials vs three-arm trials) yield different results, as well as disagreement between direct and indirect evidence. By using this approach, we will infer the presence of inconsistency from any source in the entire network on the basis of a $\mathrm{Chi}^{2}$ test. We will perform the design-by-treatment model in Stata using the 'mvmeta' command. Inconsistency and heterogeneity are interwoven; to distinguish between these two sources of variability, we will employ $\mathrm{I}^{2}$ for inconsistency to measure the percentage of variability that cannot be attributed to random error or heterogeneity (Jackson 2014).

\section{Subgroup analyses}

We will perform subgroup analyses by using the following effect modifiers as possible sources of inconsistency or heterogeneity, or both, for each primary outcome.

- Disease course (CIS, RRMS or PPMS/PRMS/SPMS).

- Previous treatment with immunotherapies (no or yes/ possible).

\section{Other sources of heterogeneity}

We will take into account the following effect modifiers by performing meta-regression or, if any, by discussing the extent to which they could alter the results/conclusions of the review.

- Age (mean or, if not provided, median).

- Gender (male vs female).

- Disease duration (mean or, if not provided, median).

\section{Sensitivity analysis}

We will perform the following sensitivity analyses for each primary outcome.
- Inclusion of only trials with low risk of attrition bias.

- Exclusion of trials with a total sample size of fewer than 50 randomised participants, to detect potential small study effects.

\section{'Summary of findings' table}

We will present the main results of this review in a 'Summary of findings' (SoF) table, according to recommendations provided in Chapter 11 of the Cochrane Handbook for Systematic Reviews of Interventions (version 5.1.0) (Schünemann 2011). We will provide estimates derived from the network meta-analysis in accordance with methods of the GRADE (Grades of Recommendation, Assessment, Development and Evaluation) Working Group (GRADE Working Group 2004). For additional details, see Salanti 2014.

We will include in the SoF table the following outcomes at longest follow-up for each drug.

- Proportion of participants who had any SAE.

- Proportion of participants who withdrew as the result of any AE.

- Proportion of participants who had a serious cardiac disorder.

- Proportion of participants who had a serious infection or infestation.

- Proportion of participants who had a serious gastrointestinal disorder.

- Proportion of participants who had a serious hepatobiliary disorder.

- Proportion of participants who had a serious immune system disorder.

For each outcome, we will choose two values for the assumed risk with placebo (i.e. second highest and second lowest placebo group risks) in the included studies. We will grade the quality of evidence for each outcome by considering study limitations, indirectness, inconsistency, imprecision of effect estimates and risk of reporting bias. According to the software GRADEpro 2008, we will assign four levels of quality of evidence: high, moderate, low and very low.

\section{Reporting}

We will report results of the review by completing the PRISMA (Preferred Reporting Items for Systematic Reviews and Meta-Analyses) harms checklist (Zorzela 2016).

\section{ACKNOWLEDGEMENTS}

We thank Andrea Fittipaldo for developing the search strategy methods used to identify studies. 


\section{R E F E R E N C E S}

\section{Additional references}

\section{Amato 2015}

Amato MP, Portaccio E. Fertility, pregnancy and childbirth in patients with multiple sclerosis: impact of diseasemodifying drugs. CNS Drugs 2015;29(3):207-20.

\section{Ascherio 2013}

Ascherio A. Environmental factors in multiple sclerosis. Expert Review of Neurotherapeutics 2013;13(12 Suppl):3-9.

Association of British Neurologists 2005

Association of British Neurologists 2005. Guidelines for the use of intravenous immunoglobulin in neurological diseases. http://www.theabn.org/documents/IVIg-Guidelines (accessed 18 April 2016). [OTHER]

\section{Atlas of MS 2013}

Multiple Sclerosis International Federation 2013. Atlas of MS 2013. Mapping multiple sclerosis around the world. http://www.msif.org/about-us/advocacy/atlas/ (accessed 18 April 2016).

Bomprezzi 2015

Bomprezzi R. Dimethyl fumarate in the treatment of relapsing-remitting multiple sclerosis: an overview. Therapeutic Advances in Neurological Disorders 2015;8(1): 20-30.

Brück 2005

Brück W. The pathology of multiple sclerosis is the result of focal inflammatory demyelination with axonal damage. Journal of Neurology 2005;252(Suppl 5):v3-9.

\section{Casetta 2007}

Casetta I, Iuliano G, Filippini G. Azathioprine for multiple sclerosis. Cochrane Database of Systematic Reviews 2007, Issue 4. [DOI: 10.1002/14651858.CD003982.pub2]

\section{Chaimani 2012}

Chaimani A, Salanti G. Using network meta-analysis to evaluate the existence of small-study effects in a network of interventions. Research Synthesis Methods 2012;3(2): $161-76$.

Chaimani 2013

Chaimani A, Higgins JP, Mavridis D, Spyridonos P, Salanti G. Graphical tools for network meta-analysis in Stata. PLoS One 2013;8(10):e76654

\section{Chouhfeh 2015}

Chouhfeh L, Kavak KS, Teter BE, Weinstock-Guttman B. Disease modifying therapies use associated with comorbid autoimmune diseases in multiple sclerosis patients. Multiple Sclerosis Related Disorded 2015;4(3):228-33.

\section{Ciccone 2008}

Ciccone A, Beretta S, Brusaferri F, Galea I, Protti A, Spreafico C. Corticosteroids for the long-term treatment in multiple sclerosis. Cochrane Database of Systematic Reviews 2008, Issue 1. [DOI: 10.1002/14651858.CD006264.pub2]

\section{Comi 2012}

Comi G, Jeffery D, Kappos L, Montalban X, Boyko A, Rocca MA, et al. Placebo-controlled trial of oral laquinimod for multiple sclerosis. New England Journal of Medicine 2012:366(11):1000-9.

\section{Compston 2008}

Compston A, Coles A. Multiple sclerosis. Lancet 2008;372: 1502-17.

\section{Confavreux 2006}

Confavreux C, Vukusic S. Natural history of multiple sclerosis: a unifying concept. Brain 2006;129(3):606-16.

\section{DerSimonian 1986}

DerSimonian R, Laird N. Meta-analysis in clinical trials. Controlled Clinical Trials 1986;7:177-88.

\section{EMA 2006}

European Agency for the Evaluation of Medicinal Products. Committee for proprietary medicinal products European public assessment report: Tysabri. http:// www.ema.europa.eu/ema/index.jsp?curl=pages/medicines/ human/medicines/000603/wapp/Post-authorisation/ human_wapp_000166.jsp\&mid=WC0b01ac058001d128 (accessed 18 April 2016).

\section{EMA 2010}

European Medicines Agency. Refusal of the marketing authorisation for Movectro (cladribine). http:// www.ema.europa.eu/docs/en_GB/document_library/ Summary_of_opinion_-_Initial_authorisation/human/ 001197/WC500101072.pdf (accessed 18 April 2016).

\section{EMA 2011}

European Medicines Agency. Committee for proprietary medicinal products European public assessment report: Gilenya. http://www.ema.europa.eu/ema/index.jsp?curl= pages/medicines/human/medicines/002202/human_med 001433.jsp\&mid=WC0b01ac058001d124 (accessed 18 April 2016).

EMA 2013a

European Medicines Agency. Committee for proprietary medicinal products European public assessment report: Aubagio. http://www.ema.europa.eu/ema/index.jsp?curl= pages/medicines/human/medicines/002514/human_med_ 001645.jsp\&mid=WC0b01ac058001d124 (accessed 18 April 2016).

\section{EMA 2013b}

European Medicines Agency. Committee for proprietary medicinal products European public assessment report: Lemtrada. http://www.ema.europa.eu/docs/en_GB/ document_library/EPAR_-_Product_Information/human/ 003718/WC500150521.pdf (accessed 18 April 2016).

\section{EMA 2014a}

European Medicines Agency. Committee for proprietary medicinal products European public assessment report: Tecfidera. http://www.ema.europa.eu/docs/en_GB/ document_library/EPAR_-_Product_Information/human/ 002601/WC500162069.pdf (accessed 18 April 2016). 
EMA 2014b

European Medicines Agency. Committee for proprietary medicinal products European public assessment report: Plegridy. http://www.ema.europa.eu/docs/en_GB/ document_library/EPAR__Summary_for_the_public/ human/002827/WC500170305.pdf (accessed 18 April 2016).

EMA 2014c

European Medicines Agency. Refusal of the marketing authorisation for Nerventra (laquinimod). http:// www.ema.europa.eu/docs/en_GB/document_library/ Summary_of_opinion_-_Initial_authorisation/human/ 002546/WC500160120.pdf (accessed 18 April 2016).

\section{EMEA 1997}

European Agency for the Evaluation of Medicinal Products. Committee for proprietary medicinal products European public assessment report: Avonex. http:// www.ema.europa.eu/docs/en_GB/document_library/ EPAR_-_Summary_for_the_public/human/000102/ WC500029423.pdf (accessed 18 April 2016).

\section{EMEA 1998}

European Agency for the Evaluation of Medicinal Products. Committee for proprietary medicinal products European public assessment report: Rebif. http:// www.ema.europa.eu/docs/en_GB/document_library/ EPAR_-_Assessment_Report_-_Variation/human/000136/ WC500122183.pdf (accessed 18 April 2016).

\section{EMEA 2002}

European Agency for the Evaluation of Medicinal Products. Committee for proprietary medicinal products European public assessment report: Betaferon. http:// www.ema.europa.eu/ema/index.jsp?curl=pages/medicines/ human/medicines/000081/human_med_000673.jsp\& mid=WC0b01ac058001d124 (accessed 18 April 2016).

Farber 2015

Farber RS, Sand IK. Optimizing the initial choice and timing of therapy in relapsing-remitting multiple sclerosis. Therapeutic Advances in Neurological Disorders 2015;8(5): 212-32.

FDA 1993

U.S. Food, Drug Administration. Betaseron interferon beta-1b subcutaneous. Drug Approval Package - Licensing Action 1993. http:// www.accessdata.fda.gov/drugsatfda_docs/label/2012/ 103471s5063s5067s5079s5088s5120s5124s5136s5138lbl.pdf (accessed 18 April 2016).

\section{FDA 1996}

U.S. Food, Drug Administration. Glatiramer acetate (Capoxane) Product Approval Information - Licensing Action 1996. http://www.accessdata.fda.gov/scripts/ cder/drugsatfda/index.cfm?fuseaction=Search.Label_ ApprovalHistory\#apphist (accessed 18 April 2016).

\section{FDA 2000}

U.S. Food, Drug Administration. Mitoxantrone (Novantrone) Product Approval Information - Licensing Action 2000. http://www.accessdata.fda.gov/drugsatfda_ docs/label/2009/019297s030s031lbl.pdf (accessed 18 April 2016).

FDA 2002

U.S. Food, Drug Administration. Interferon beta-1a (Rebif) Product Approval Information - Licensing Action 2002. http://www.fda.gov/Drugs/DevelopmentApprovalProcess/ HowDrugsareDevelopedandApproved/ ApprovalApplications/TherapeuticBiologicApplications/ ucm080737.htm (accessed 18 April 2016).

\section{FDA 2003}

U.S. Food, Drug Administration. Interferon beta-1a (Avonex) Product Approval Information - Licensing Action 2003. http://www.accessdata.fda.gov/drugsatfda_docs/nda/ 2003/103628s5021TOC.cfm (accessed 18 April 2016).

\section{FDA 2006}

U.S. Food, Drug Administration. FDA Approves Resumed Marketing of Tysabri Under a Special Distribution Program. http://www.fda.gov/NewsEvents/Newsroom/ PressAnnouncements/2006/ucm108662.htm (accessed 18 April 2016).

\section{FDA 2010}

U.S. Food, Drug Administration. Gilenya (Fingolimod) Product Approval Information 2010. http:// www.accessdata.fda.gov/scripts/cder/drugsatfda/index.cfm? fuseaction=Search.Set_Current_Drug\&ApplNo=022527\& DrugName=GILENYA $\&$ ActiveIngred $=$ FINGOLIMOD $\&$ SponsorApplicant=NOVARTIS\&ProductMktStatus $=1 \&$ goto=Search.DrugDetails (accessed 18 April 2016).

\section{FDA 2012}

U.S. Food, Drug Administration. Aubagio (Teriflunomide) Product Approval Information 2012. http://www.accessdata.fda.gov/scripts/cder/ drugsatfda/index.cfm?fuseaction=Search.Set_Current Drug\&ApplNo=202992 $\&$ DrugName=AUBAGIO $\&$ ActiveIngred $=$ TERIFLUNOMIDE $\&$ SponsorApplicant $=$ SANOFI\%20AVENTIS\%20US\&ProductMktStatus=1 \& goto=Search.DrugDetails (accessed 18 April 2016).

\section{FDA 2013a}

U.S. Food, Drug Administration. Tecfidera (Dimethyl fumarate) Product Approval Information 2013. http:// www.accessdata.fda.gov/scripts/cder/drugsatfda/index.cfm? fuseaction=Search.Set_Current_Drug\&ApplNo= 204063\&DrugName $=$ TECFIDERA $\&$ ActiveIngred $=$ DIMETHYL\%20FUMARATE $\&$ SponsorApplicant= BIOGEN\%20IDEC\%20INC\&ProductMktStatus=1\& goto=Search.DrugDetails (accessed 18 April 2016).

\section{FDA 2014a}

U.S Food, Drug Administration. Alemtuzumab (Lemtrada) Product Approval Information. Licensing Action 2014. http://www.accessdata.fda.gov/drugsatfda_docs/appletter/ 2014/103948Orig1s5139ltr.pdf (accessed 18 April 2016).

\section{FDA 2014b}

U.S. Food, Drug Administration. Peginterferon beta1a (Plegridy) Product Approval Information. Licensing Action 2014. https://www.plegridy.com/content/dam/ 
commercial/multiple-sclerosis/plegridy/pat/en_us/pdf/ november/plegridy-prescribing-information.pdf (accessed 18 April 2016).

\section{Federal Register 2015}

Content and format of labeling for human prescription drug and biological products; requirements for pregnancy and lactation labeling. https://www.federalregister.gov/ articles/2014/12/04/2014-28241/content-and-formatof-labeling-for-human-prescription-drug-and-biologicalproducts-requirements-for (accessed 18 April 2016).

\section{Friese 2014}

Friese MA, Schattling B, Fugger L. Mechanisms of neurodegeneration and axonal dysfunction in multiple sclerosis. Nature Reviews. Neurology 2014;10(4):225-38.

Giovannoni 2010

Giovannoni G, Comi G, Cook S, Rammohan K, Rieckmann P, Soelberg Sørensen P, et al. A placebocontrolled trial of oral cladribine for relapsing multiple sclerosis. New England Journal of Medicine 2010;362(5): 416-26.

Goodin 2002

Goodin DS, Frohman EM, Garmany GP Jr, Halper J, Likosky WH, Lublin FD, et al. Disease modifying therapies in multiple sclerosis: report of the Therapeutics and Technology Assessment Subcommittee of the American Academy of Neurology and the MS Council for Clinical Practice Guidelines. Neurology 2002;58(2):169-78.

\section{Goodkin 1996}

Goodkin DE, Rudick RA, VanderBrug Medendorp S, Daughtry MM, Van Dyke C. Low-dose oral methotrexate in chronic progressive multiple sclerosis: analyses of serial MRIs. Neurology 1996;47(5):1153-7.

\section{Goodman 2006}

Goodman LS, Gilman A, Brunton LL, Lazo JS, Parker KL. Goodman \& Gilman's the pharmacological basis of therapeutics. New York: McGraw-Hill, 2006.

\section{GRADE Working Group 2004}

GRADE Working Group. Grading quality of evidence and strength of recommendations. British Medical Journal 2004; 328:1490-4.

\section{GRADEpro 2008 [Computer program]}

Brozek J, Oxman A, Schünemann H. GRADEpro Version 3.2 for Windows. Brozek J, Oxman A, Schünemann H, 2008.

\section{Gray 2004}

Gray O, McDonnell GV, Forbes RB. Methotrexate for multiple sclerosis. Cochrane Database of Systematic Reviews 2004, Issue 2. [DOI: 10.1002/14651858.CD003208.pub2]

Hauser 2008

Hauser SL, Waubant E, Arnold DL, Vollmer T, Antel J, Fox RJ, et al. B-cell depletion with rituximab in relapsingremitting multiple sclerosis. New England Journal of Medicine 2008;358(7):676-88.

\section{Hawker 2009}

Hawker K, O'Connor P, Freedman MS, Calabresi PA, Antel J, Simon J, et al. Rituximab in patients with primary progressive multiple sclerosis: results of a randomized double-blind placebo-controlled multicenter trial. Annals of Neurology 2009;66(4):460-71.

\section{Higgins 2011}

Higgins J, Green S (editors). Cochrane Handbook for Systematic Reviews of Interventions Version 5.1.0 [updated March 2011]. The Cochrane Collaboration, 2011. www.cochrane-handbook.org.

\section{Higgins 2012}

Higgins JPT, Jackson D, Barrett JK, Lu G, Ades AE, White IR. Consistency and inconsistency in network metaanalysis: concepts and models for multi-arm studies. Research Synthesis Methods 2012;3:98-110.

\section{Hollenbach 2015}

Hollenbach JA, Oksenberg JR. The immunogenetics of multiple sclerosis: a comprehensive review. Journal of Autoimmunity 2015;64:13-25.

Hutchinson 2015

Hutchinson M. Neurodegeneration in multiple sclerosis is a process separate from inflammation: no. Multiple Sclerosis (Houndmills, Basingstoke, England) 2015;21(13):1628-31.

\section{ICH 2015}

International Conference on Harmonisation of Technical Requirements for Registration of Pharmaceuticals for Human Use (ICH). Introductory guide MedDRA version 18.0. http://www.meddra.org/sites/default/files/guidance/ file/intguide_18_0_english.pdf (accessed 18 April 2016).

\section{Jackson 2014}

Jackson D, Barrett JK, Rice S, White IR, Higgins JP. A design-by-treatment interaction model for network metaanalysis with random inconsistency effects. Statistics in Medicine 2014;33(21):3639-54.

\section{Kappos 2011}

Kappos L, Li D, Calabresi PA, O’Connor P, Bar-Or A, Barkhof $F$, et al. Ocrelizumab in relapsing-remitting multiple sclerosis: a phase 2, randomised, placebocontrolled, multicentre trial. Lancet 2011;378(9805): 1779-87.

\section{Kremenchutzky 2006}

Kremenchutzky M, Rice GP, Baskerville J, Wingerchuk DM, Ebers GC. The natural history of multiple sclerosis: a geographically based study 9: observations on the progressive phase of the disease. Brain 2006;129(Pt 3): 584-94.

\section{La Mantia 2007}

La Mantia L, Milanese C, Mascoli N, D'Amico R, Weinstock-Guttman B. Cyclophosphamide for multiple sclerosis. Cochrane Database of Systematic Reviews 2007, Issue 1. [DOI: 10.1002/14651858.CD002819.pub2]

\section{Leist 2014}

Leist TP, Comi G, Cree BA, Coyle PK, Freedman MS Hartung HP, et al. Effect of oral cladribine on time to conversion to clinically definite multiple sclerosis in patients with a first demyelinating event (ORACLE MS): a phase 3 randomised trial. Lancet Neurol 2014;13(3):257-67. 


\section{Louapre 2015}

Louapre C, Lubetzki C. Neurodegeneration in multiple sclerosis is a process separate from inflammation: yes. Multiple Sclerosis (Houndmills, Basingstoke, England) 2015; 21(13):1626-8.

\section{Lublin 1996}

Lublin F, Reingold S. Defining the clinical course of multiple sclerosis: results of an international survey. National Multiple Sclerosis Society (USA) Advisory Committee on Clinical Trials of New Agents in Multiple Sclerosis. Neurology 1996;46:907-11.

\section{Lublin 2014}

Lublin FD, Reingold SC, Cohen JA, Cutter GR, Sørensen PS, Thompson AJ, et al. Defining the clinical course of multiple sclerosis: the 2013 revisions. Neurology 2014;83 (3):278-86.

\section{Lycke 2015}

Lycke J. Monoclonal antibody therapies for the treatment of relapsing-remitting multiple sclerosis: differentiating mechanisms and clinical outcomes. Therapeutic Advances in Neurological Disorders 2015;8(6):274-93.

\section{Martinelli-Boneschi 2005}

Martinelli Boneschi F, Vacchi L, Rovaris M, Capra R, Comi G. Mitoxantrone for multiple sclerosis. Cochrane Database of Systematic Reviews 2013, Issue 5. [DOI: 10.1002/ 14651858.CD002127.pub3]

\section{Martìnez-Càceres 1998}

Martínez-Cáceres EM, Río J, Barrau M, Durán I, Borrás C, Tintoré $\mathrm{M}$, et al. Amelioration of flulike symptoms at the onset of interferon beta-1b therapy in multiple sclerosis by low-dose oral steroids is related to a decrease in interleukin6 induction. Annals of Neurology 1998;44(4):682-5.

\section{McDonald 2001}

McDonald W, Compston A, Edan G, Goodkin D, Hartung $\mathrm{H}$, Lublin F. Recommended diagnostic criteria for multiple sclerosis: guidelines from the international panel on the diagnosis of multiple sclerosis. Annals of Neurology 2001; 50:121-7.

\section{Miladinovic 2014}

Miladinovic B, Hozo I, Chaimani A, Djulbegovic B. Indirect treatment comparison. Stata Journal 2014;14(1): 76-86.

Multiple-Treatments Meta-analysis (MTM) Multiple-Treatments Meta-analysis (MTM). A framework for evaluating and ranking multiple healthcare technologies. http://www.mtm.uoi.gr/ (accessed 18 April 2016).

\section{Murphy 2010}

Murphy JA, Harris JA, Crannage AJ. Potential short-term use of oral cladribine in treatment of relapsing-remitting multiple sclerosis. Neuropsychiatric Disease and Treatment 2010;6:619-25.

Pakpoor 2015

Pakpoor J, Disanto G, Altmann DR, Pavitt S, Turner BP, Marta M, et al. No evidence for higher risk of cancer in patients with multiple sclerosis taking cladribine. Neurology Neuroimmunology \& Neuroinflammation 2015;2(6):e158.

\section{Peters 2008}

Peters J, Sutton A, Jones D, Abrams K, Rushton L. Contourenhanced meta-analysis funnel plots help distinguish publication bias from other causes of asymmetry. Journal of Clinical Epidemiology 2008;61(10):991-6.

Polman 2005

Polman C, Reingold S, Edan G, Filippi M, Hartung H, Kappos L, et al. Diagnostic criteria for multiple sclerosis: 2005 revisions to the 'McDonald Criteria'. Annals of Neurology 2005;58:840-6.

Polman 2011

Polman CH, Reingold SC, Banwell B, Clanet M, Cohen JA, Filippi M, et al. Diagnostic criteria for multiple sclerosis: 2010 revisions to the McDonald criteria. Annals of Neurology 2011;69:292-302.

Poser 1983

Poser C, Paty D, Scheinberg L, McDonald W, Davis F, Ebers G, et al. New diagnostic criteria for multiple sclerosis: guidelines for research protocols. Annals of Neurology 1983; 13:227-31.

\section{Salanti 2011}

Salanti G, Ades A, Ioannidis J. Graphical methods and numerical summaries for presenting results from multipletreatment meta-analysis: an overview and tutorial. Journal of Clinical Epidemiology 2011;64:163-71.

\section{Salanti 2012}

Salanti G. Indirect and mixed-treatment comparison, network, or multiple-treatments meta-analysis: many names, many benefits, many concerns for the next generation evidence synthesis tool. Research Synthesis Methods 2012;3(2):80-97.

\section{Salanti 2014}

Salanti G, Del Giovane C, Chaimani A, Caldwell DM, Higgins JP. Evaluating the quality of evidence from a network meta-analysis. PLoS One 2014;9:e99682.

\section{Scalfari 2010}

Scalfari A, Neuhaus A, Degenhardt A, Rice GP, Muraro PA Daumer $\mathrm{M}$, et al. The natural history of multiple sclerosis: a geographically based study 10: relapses and long-term disability. Brain 2010;133(Pt 7):1914-29.

\section{Scalfari 2013}

Scalfari A, Knappertz V, Cutter G, Goodin DS, Ashton R, Ebers GC. Mortality in patients with multiple sclerosis. Neurology 2013;81(2):184-92.

\section{Scalfari 2014}

Scalfari A, Neuhaus A, Daumer M, Muraro PA, Ebers GC. Onset of secondary progressive phase and longterm evolution of multiple sclerosis. Journal of Neurology, Neurosurgery \& Psychiatry 2014;85:67-75.

\section{Schünemann 2011}

Schünemann H, Oxman A, Higgins J, Vist G, Glasziou P, Guyatt G. Chapter 11: Presenting results and 'Summary of findings' tables. In: Higgins JPT, Green $S$ (editors). Cochrane Handbook for Systematic Reviews of Interventions Version 5.1.0 [updated March 2011]. 
The Cochrane Collaboration, 2011. www.cochrane-

handbook.org.

\section{Simpson 2015}

Simpson S Jr, Taylor BV, van der Mei I. The role of epidemiology in MS research: past successes, current challenges and future potential. Multiple Sclerosis

(Houndmills, Basingstoke, England) 2015;21(8):969-77.

Van Assche 2005

Van Assche G, Van Ranst M, Sciot R, Dubois B, Vermeire S, Noman M, et al. Progressive multifocal leukoencephalopathy after natalizumab therapy for Crohn's disease. New England Journal of Medicine 2005;353(4): 362-8.

Veroniki 2013

Veroniki AA, Vasiliadis HS, Higgins JP, Salanti G. Evaluation of inconsistency in networks of interventions. Internal Journal Epidemiology 2013;42(1):332-45.

Vollmer 2014

Vollmer TL, Sorensen PS, Selmaj K, Zipp F, Havrdova E, Cohen JA, et al. A randomized placebo-controlled phase III trial of oral laquinimod for multiple sclerosis. Journal of Neurology 2014;261(4):773-83.
Weinshenker 1989

Weinshenker BG, Bass B, Rice GP, Noseworthy J, Carriere W, Baskerville J, et al. The natural history of multiple sclerosis: a geographically based study. I. Clinical course and disability. Brain 1989;112(Pt 1):133-46.

White 2011

White IR. Multivariate random-effects meta-regression: updates to mvmeta. The STATA Journal 2011;11:255-70.

White 2012

White IR, Barrett JK, Jackson D, Higgins JPT. Consistency and inconsistency in network meta-analysis: model estimation using multivariate meta-regression. Research Synthesis Methods 2012;3(2):111-25.

Williamson 2015

Williamson EM, Berger JR. Central nervous system infections with immunomodulatory therapies. Continuum (Minneapolis Minn.) 2015;21(6 Neuroinfectious Disease): 1577-98.

\section{Zorzela 2016}

Zorzela L, Loke YK, Ioannidis JP, Golder S, Santaguida P, Altman DG, et al. PRISMA harms checklist: improving harms reporting in systematic reviews. BMJ 2016;352:1157.

* Indicates the major publication for the study

\section{A P P E N D I C E S}

\section{Appendix I. Keywords for searching the MS Group Register}

\{interferon $1^{*}$ \} OR \{interferon beta\} OR \{beta-1 interferon\} OR \{beta 1 interferon\} OR \{interferon beta- $\left.11^{*}\right\}$ OR \{rebif\} OR \{avonex\}

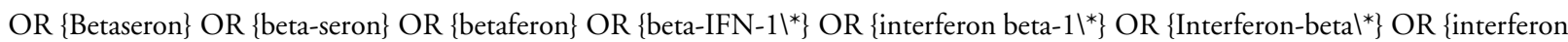
betal*\} OR \{recombinant interferon beta-1 $\left.1^{*}\right\}$ OR $\{$ beta-1a interferon\} OR \{beta 1a interferon\} OR \{interferon beta-1a\} OR \{beta $1 \mathrm{~b}$ interferon\} OR \{interferon beta1b \} OR \{IFNb-1b\} OR \{IFNbeta-1b\} OR \{interferon beta-1b\} OR \{novantrone\} OR \{novantron\} OR \{onkotrone\} OR \{pralifan\} OR \{mitozantrone\} OR \{mitoxantrone\} OR \{copolymer-1\} OR \{cop-1\} OR \{copaxone\} OR \{glatiramer acetate OR $\{$ cpx $\}$ OR \{cop1\} OR \{copolymer\} OR \{glatiramer\} OR \{immunomodulation $\left.\left.\right|^{*}\right\}$ OR \{immunomodulator $\left.{ }^{*}\right\}$ OR \{immunosuppression\} OR \{antegren\} OR \{natalizumab OR \{tysabri\} OR \{monoclonal antibody*\} OR \{Antibodies, Monoclonal\} OR \{fingolimod\} OR \{FTY720\} OR \{FTY 720\} OR \{fingolimod hydrochloride OR \{FTY-720\} OR \{2-amino-2-(2-(4-octylphenyl)ethyl)1,3-propanediol hydrochloride\} OR \{Gilenya\} OR \{sphingosine-fosphate-receptor antagonist\} OR \{HMR1726\} OR \{A77 1726\} OR \{Leflunomide\} OR \{Arava\} OR \{teriflunomide\} OR \{TFN\} OR \{teriflunomide-D4\} OR \{A771726\} OR \{Dihydroorotate dehydrogenase (DHODH) inhibitors $\}$ OR \{(Z)-2-Cyano-3-hydroxy-N-[4-(trifluoromethyl)phenyl]-2-butenamide $\}$ OR \{dimethyl lfumarate OR \{Fumaderm $\}$ OR \{FAG 201\} OR \{FAG201\} OR \{FAG-201\} OR \{BG 00012\} OR \{BG00012\} OR \{BG-00012\} OR \{BG 12 compound OR \{BG12 compound\} OR \{BG-12 compound\} OR \{BG-12\} OR \{tecfidera\} OR \{Nrf2 activator\} OR \{oral fumarate\} OR \{fumaric acid eaters\} OR \{alemtuzumab OR \{Campath 1G\} OR \{Campath-1G\} OR \{Campath-1-G\} OR \{Campath 1M\} OR \{Campath-1M\} OR \{MabCampath\} OR \{Schering brand of alemtuzumab\} OR \{Campath\} OR \{Berlex brand of alemtuzumab\} OR \{Campath 1H\} OR \{monoclonal antibody Campath-1H\} OR \{Campath-1H\} OR \{monoclonal antibody*\} OR \{Antibodies, Monoclonal\} OR \{lemtrada\} OR \{daclizumab\} OR \{antigen\} OR \{zenapax\} OR \{dacliximab\} OR \{monoclonal antibody\} OR \{monoclonal antibodies\} OR \{antigens\} OR \{Laquinimod\} OR \{azathioprine\} OR \{azathioprine\} OR \{immuran\} OR \{imuran\} OR \{imurel\} OR $\left\{\right.$ immunoglobulin $\left.1^{*}\right\}$ OR $\left\{\right.$ intravenous immunoglobulin $\left.1^{*}\right\}$ OR $\left\{\mathrm{iV}\right.$ immunoglobulin $\left.\left.\right|^{*}\right\}$ \{intravenous $\}$ OR $\{$ Intravenous IG $\}$ OR $\{$ Intravenous Antibodies\} \{ivig\} OR \{igiv\} OR \{adrenal cortex hormones\} OR \{steroid $\left.{ }^{*}\right\}$ OR \{methylprednisolone\} OR \{prednisolone\} OR \{dexamethasone\} OR \{corticosteroidl*\} OR \{acth\} OR \{prednisone\} OR \{Adrenocorticotropic Hormone\} OR \{polyethylene glycol- 
interferon-beta-1a\} OR \{PEG IFN-beta-1a\} OR \{Pegylated interferon beta-1a\} OR \{Ocrelizumab\} OR \{placebol*\} OR \{12-Chloro-2'deoxyadenosine OR \{2-Chlorodeoxyadenosine OR \{2'-Deoxy-2-chloroadenosine OR \{Leustatin\} OR \{methotrexate OR \{mexate OR \{dicesium salt methotrexate\} OR \{disodium salt methotrexate\} OR \{sodium salt methotrexate\} OR \{methotrexate hydrate\} OR \{(D)-isomer methotrexate OR $\{(\mathrm{DL})$-isomer methotrexate\} OR \{cyclophosphamide\} OR \{phosphoramide mustard $\backslash\}$ OR $\backslash\{\mathrm{cfx}\}$ OR \{cyclophosphane\} OR \{cytophosphan\} OR \{Cyclophosphamide Monohydrate\} OR \{(+-)-Isomer Cyclophosphamide\} OR \{phosphoramide mustards\}

\title{
CONTRIBUTIONSOFAUTHORS
}

Concept development - AC, CDG, GF, IT, LLM, LM, MDB.

Title registration - All.

Drafting of protocol - AC, IT, LLM, MDB.

Editing of protocol - All.

\section{DECLARATIONSOF INTEREST}

\author{
MDB - none. \\ AC - none. \\ IT - none. \\ SF - none. \\ LLM - none. \\ GC - none. \\ MC - none. \\ MGL - none. \\ CDG - none. \\ $\mathrm{SN}$ - none. \\ LM - none.
}

GF - none. As Co-ordinating Editor, Dr. Filippini was excluded from the editorial process to ensure separation of the review author from the editorial process. This includes all editorial decisions and related activities (e.g. sign-off for publication).

\section{SOURCES OF SUPPORT}




\section{Internal sources}

- Fondazione I.R.C.C.S. Istituto Neurologico Carlo Besta, Milan, Italy.

\section{External sources}

- Ministero della Salute, Italy. 